\title{
La digitalización de la televisión pública europea: la estrategia de la RAI
}

David Álvarez Rivas - Universidad Complutense de Madrid - dalvarez@ucm.es María José Pérez del Pozo - Universidad Complutense de Madrid mjperezp@ucm.es

Sergio Príncipe Hermoso - Universidad Complutense de Madrid sprincip@ucm.es

\section{Resumen:}

La Radiotelevisión pública italiana RAI ha representado históricamente, en el ámbito de la Europa comunitaria, un modelo público peculiar debido a su fuerte condicionamiento de una política nacional convulsa e inestable. Ese carácter sui géneris ha estado presente también en el proceso de digitalización de la cadena iniciada con la Ley 66/01 y la controvertida "Ley Gasparri" de 2004, que crearon mecanismos de protección para la RAI -y la privada Mediaset- frente a la prevista avalancha de nuevos operadores y plataformas; a la vez, nuevos marcos legales en 2015 y 2017 van concretando la función de servicio audiovisual público que debe regir las actividades de la cadena.

Todo el complejo -y, a veces, contradictorio- entramado legal en torno a la RAI ha afectado a su desarrollo y expansión digitales, al cierre de su etapa analógica y al inicio de la TDT. Sin embargo, y pese a las interferencias y peajes políticos que contaminan en ocasiones su mandato de servicio público, vive un proceso de adaptación y diversificación de contenidos, creación de nuevos formatos para nuevos públicos y desarrollo de proyectos de innovación, investigación para adaptarse al Mercado Único Europeo, y competir con un sector privado y de pago que siempre ha liderado los datos de audiencia. 


\section{Las nuevas narrativas, en el entorno social \\ Universidad de La Laguna, diciembre de 2019}

A lo largo de este trabajo se analiza la estrategia digital seguida por la RAI en los últimos años, desde una perspectiva descriptiva, para poder evaluar si existe realmente un plan estratégico de transformación de los servicios digitales y de acceso y participación de los espectadores, así como nuevos mecanismos y recursos de transparencia propios de iniciativas política de servicio público.

Palabras clave: Televisión Pública Italiana; Mercado Único Digital; innovación; digitalización; adaptación; contenidos.

\section{Introducción}

Acercarse al panorama televisivo italiano es siempre una tarea compleja dado que las relaciones entre el poder político, el mundo de los negocios y los medios de comunicación están estrechamente ligados entre sí. Consecuencia de este peculiar escenario es que no siempre hay un trasvase directo entre lo que se fija en el marco normativo y el desarrollo real y concreto de las políticas en materia audiovisual. En cualquier caso, el punto de partida para todo análisis en profundidad nos lleva a individualizar los dos grandes colosos audiovisuales que operan en Italia: el sistema audiovisual público Rai, y el conglomerado mediático, privado y comercial -en definitiva, el imperio empresarial en manos de la familia Berlusconi-, Mediaset. La relación entre ambos ha sido determinante en el mercado audiovisual italiano, reafirmando sus posiciones ante procesos clave como la digitalización de la señal y el surgimiento de nuevos agentes y operadores que han tratado de abrirse camino en medio de esta gran polarización televisiva.

La base fundamental para la digitalización de la televisión en Italia es la Ley 66/01 (Gazzetta Ufficiale, 2001) que fija para 2006 el apagado de los sistemas analógicos. El proceso estuvo plagado de tantos trámites parlamentarios, polémicas, retrasos técnicos y modificaciones legales que no pudo completarse hasta seis años después. En este sentido, no contribuyó a aclarar el panorama la Ley 112/04 (Gazzetta Ufficiale, 2004), conocida como "Ley Gasparri", denominada así porque la impulsó el ministro italiano para las Comunicaciones, Maurizio Gasparri, bajo mandato del entonces primer ministro, Silvio Berlusconi. 


\section{Las nuevas narrativas, en el entorno social \\ Universidad de La Laguna, diciembre de 2019}

Dicha Ley fue polémica en sí misma por múltiples motivos, pero gran parte de las interpretaciones a las que esta normativa se veía sometida dejaban entrever dos circunstancias 0 , mejor dicho, intenciones políticas, sobre la televisión en Italia. Por un lado, trataba de establecerse un nuevo modelo de gestión de la Rai que, lejos de simplificar su gestión, más bien afirmaba su dependencia estatal bajo el reclamo del "servicio público". Por otro, esta propuesta normativa parecía blindar a Mediaset, a la sazón, imprescindible eje empresarial de Silvio Berlusconi, frente a las amenazas que podían suponer otros operadores y las nuevas formas de hacer y transmitir televisión que venían de la mano del salto digital. Sin entrar en un análisis detallado, la Ley Gasparri planteaba -entre otros particulares técnicos- que en el reparto de las nuevas frecuencias que se derivaría de la digitalización de la señal, los grandes operadores analógicos debían tener, no solo la prioridad, sino también la posibilidad de posicionar, ampliar su estructura, y, por tanto, proyectar nuevas estrategias de contenidos. La idea era la de ir avanzando y asegurando, de manera progresiva, la infraestructura necesaria y ofrecer, en tiempo y forma, contenidos distribuidos en señal digital íntegra y en abierto. Pero esta propuesta generó incertidumbres porque podría provocar un modelo que privilegiaba estas transmisiones en ausencia de equilibrios. Los riesgos para una libre competencia audiovisual eran más que evidentes y así se lo apercibió la propia Comisión Europea (2006) en una carta formal al Gobierno italiano en la que subrayaba que la Ley 112/04 "permite a los operadores incumbentes mantener el control sobre las frecuencias y las redes incluso antes del apagón analógico, de tal modo que se limita las capacidades, por parte de los competidores, de disfrutar y consolidarse en las redes digitales".

En medio de polémicas y decretos que iban reformando las lagunas legislativas de los marcos normativos de referencia, Italia se vio obligada a retrasar el encendido digital en dos ocasiones. Finalmente, tras una implantación progresiva en diferentes regiones del país ${ }^{1}$, el apagón analógico definitivo se concretó el 4 de julio de 2012.

1 Los primeros apagones se produjeron en el último trimestre de 2009. 


\section{Las nuevas narrativas, en el entorno social}

\section{Universidad de La Laguna, diciembre de 2019}

En todo este proceso, todos los operadores implicados aunaron intereses y esfuerzos en la puesta en marcha en 2008 de TIVÚ $^{2}$, una plataforma que aglutinaba a Rai, Mediaset y la compañía de telecomunicaciones italiana, Telecom Italia, con la misión de promover los servicios digitales y diseñar una parrilla de contenidos íntegramente digital.

Una vez que la TDT se ha consolidado en el país, el escenario de referencia del audiovisual público italiano se ve circundado por un tejido televisivo que, según datos de la sección de radio y televisión de Confindustria ${ }^{3}$, la confederación empresarial italiana, contabilizaba a cierre de 2017 de 423 canales de televisión, 356 de ellos de marca nacional pues su base de producción y emisión depende de editores italianos.

Los datos que ofrece Confindustria ofrecen otras lecturas interesantes. Por ejemplo, en 2017 se constata que en Italia se concentran más canales de televisión en el satélite (287) que en la TDT (123). Y en la comparación, siempre cuantitativa en este caso, las plataformas de pago cuentan con más oferta (232 canales) que las cadenas de libre acceso y gratuitas (124). Pero detengámonos en este último aspecto, ya que las televisiones aglutinadas en TIVÚ, la sociedad que se instituyó para el desarrollo de la transición digital en Italia gracias a la acción conjunta de Rai, Mediaset y Telecom Italia, representan el 56,4\%. Por tanto, como conclusión previa se pueda extraer que, en el marco de la consolidación de la televisión digital terrestre desde el apagón analógico en el país, han predominado las plataformas de pago frente a las propuestas gratuitas. Entre estas, las televisiones (Rai y Mediaset) que ya eran actores relevantes en el contexto analógico son las más extendidas y las que mayor cobertura ofrecen.

Por tanto, ¿cuál es el lugar de Rai en esta aproximación? Si nos fijamos en el análisis de Confindustria, Rai mantiene 26 canales en la red TDT, los mismos que reproduce a través del satélite, y por supuesto, gratuitos. Sin embargo, con esta estructura, la radiotelevisión pública del país se ve superada por la

\footnotetext{
2 Consúltese su web institucional: https://www.tivu.tv/tivu-srl.html

3 Para un desarrollo más prolijo, es muy útil consultar los informes sectoriales de Confindustria aunque se centran, como es lógico, en los canales privados: http://confindustriaradiotv.it/canali-tv-italia-2017/
} 
pujanza de Mediaset cuya oferta de canales (tanto de pago como de libre acceso) dobla a la del modelo público.

En este contexto, no conviene tampoco alejarse de la nueva tendencia que en el mercado italiano está irrumpiendo en consonancia con otros países europeos. La irrupción de las plataformas de vídeo en 'streaming' están logrando convertirse en alternativa para el consumo audiovisual de los italianos. Según refiere Biondi (2018), en un informe publicado por la consultora EY se pone de manifiesto que en el sector de la televisión de pago y a demanda, los grupos Sky y Mediaset -en concreto su versión Premium- siguen liderando, acaparando 6,5 millones de usuarios. Las plataformas consideradas 'over the top' (OTT) como Netflix y otros rivales acumulan una base de clientes conjunta de 5 millones. Lo significativo, tal y como subraya el análisis de EY al que alude Biondi, es que de 2017 a 2018 el impulso de las plataformas ha experimentado una escalada explosiva ya que partía de una cuota de mercado de 2,3 millones de usuarios. En el reporte informativo de Biondi se hace hincapié en que EY no ofrece datos disgregados, pero todo apunta a que Netflix es la plataforma que más éxito de audiencia ha tenido muy por encima de Amazon Prime Video y otras alternativas.

Especialmente interesante es observar, en paralelo, el consumo audiovisual a través de internet de modo gratuito. La plataforma digital de la pública Rai (Raiplay), la análoga del grupo Mediaset Play y el gigante YouTube aúnan, en conjunto, a 20,9 millones de italianos que optan por esta vía para disfrutar de contenidos televisivos.

\section{La RAI en el nuevo escenario digital}

En ocasiones, punto de referencia; en otras, institución indispensable y de bandera, y no pocas veces blanco de las críticas de la audiencia italiana, Rai no solo es la salvaguarda de gran parte del patrimonio audiovisual italiano, sino también parte de su historia. Las primeras emisiones de la televisión estatal italiana tienen lugar el 3 de enero de $1954^{4}$ mientras que el arranque de la radio surgió en la década de los años 20 del siglo pasado. De este modo, Italia

\footnotetext{
4 Véase web institucional de Rai para estos pormenores: http://www.rai.it/dl/rai/text/Contentltem20844e48-74d8-44fe-a6f47c224c96e8e4.html?refresh ce
} 


\section{Las nuevas narrativas, en el entorno social \\ Universidad de La Laguna, diciembre de 2019}

comparte con muchos países europeos una cronología similar a la hora de poner en marcha la espina dorsal de sus medios audiovisuales, primero estatales, luego públicos.

La integración de los tres programas de radio y la única cadena de televisión que había en el momento se llevó a cabo de modo paulatino pero natural. Poco a poco, Rai iba fortaleciéndose hasta que en 1962 se inauguraron las emisiones de la segunda cadena. El tercer canal de la pública italiana llegará bordeando los años 80 , cuando ya Silvio Berlusconi trataba de abrirse camino en el sector televisivo comercial con la entonces pequeña y local Telemilano.

Con esta estructura de tres cadenas de televisión de ámbito nacional, aunque si bien el tercer canal servía en gran medida de acomodo a los contenidos más regionales, y tres grandes emisoras de radio, la Rai se mantuvo estable hasta, prácticamente, su salto al siglo XXI. Más espacio en el contexto analógico no podía haber para que Rai creciese, más allá de inaugurar, vía web, su presencia en internet en un prematuro 1996. Los resortes -lentos y confusos en una primera fase- que perfilaron el futuro digital de la televisión italiana comenzarían a pergeñarse en 2003, aunque el proceso no se completó hasta 2012. El salto paulatino de Rai hacia la TDT se tradujo en un incremento importantísimo de la oferta televisiva. Además, este hecho supuso una novedad para el modelo público italiano ya que permitió el impulso de nuevos formatos y propuestas diversificadas y específicas. No en vano, pese a contar con tres cadenas base, ninguna de ellas se había tematizado o especializado antes de la digitalización de la señal, sino que de manera insólita Rai1, Rai2 y Rai3 habían convivido como televisiones generalistas. Las únicas diferencias entre ellas se basaban, fundamentalmente, en la línea editorial de sus informativos y en el público objetivo. Bien es verdad que, de todas ellas, Rai3 había tenido un rol algo diferente pues su apuesta por programar contenidos más relacionados con el ámbito regional y cultural 0 , incluso, filosófico y de mayor profundidad, la convertían en una cadena algo peculiar en comparación con sus "hermanas".

¿Pero cuál ha sido el estatus jurídico de Rai, más allá de ser considerada una institución tan básica como a veces criticada en el contexto italiano? De nuevo, la clave la ofrece la Ley Gasparri de 2004, que configurará el modelo 
audiovisual público italiano como una "società per azioni", es decir, una sociedad anónima en la que el Estado asume el rol de principal e imprescindible accionista.

Desde entonces, poco ha cambiado en este sentido el perfil de Rai. Donde sí ha habido modificaciones, o mejor dicho, ampliaciones, es en la perspectiva de los objetivos del conglomerado público italiano. A partir de un decreto aprobado por el Gobierno italiano en 2017, se matiza que Rai asume en exclusiva la concesión por una década del servicio audiovisual público y multimedia.

Con respecto a su estructura, Rai presenta un modelo que podemos denominar "centralizado", pues desde una única corporación se asumen las diversas facetas que configuran la misión de la radiotelevisión pública en Italia. En su organigrama interno, la radiotelevisión pública italiana se divide en las siguientes áreas:

- Dirección editorial y de servicios informativos: Desde esta sección se coordinan las distintas cadenas de radio y televisión de Rai de carácter generalista, y las distintas propuestas temáticas que se subdividen en Rai Fiction (entretenimiento: cine y series), Rai Gold (contenidos destinados a una audiencia adulta), Rai Ragazzi (contenidos para público infantil y juvenil) y Rai Cultura. Lo que llama la atención de esta propuesta es que en el organigrama aparecen desgajados de las distintas cadenas que componen la oferta de Rai, los servicios informativos para Rai 1, Rai 2 y Rai 3, así como los telediarios regionales y deportivos. Parece, pues, que el eje informativo tiene su propia entidad específica en sus distintas versiones sin que exista una coordinación única que aglutine a todas ellas.

- Dirección Financiera, encargada del balance económico y la misión administrativa de la radiotelevisión pública.

- Dirección Corporativa y de Soporte: Asume los recursos humanos de Rai.

- Dirección Tecnológica: Además de la infraestructura tecnológica de Rai, gestiona los bienes inmobiliarios, aspecto también curioso.

- Dirección Digital: Diseña la oferta y los contenidos digitales de la radiotelevisión. 
- Dirección operativa: Su misión es la de desarrollar la oferta de producción televisiva.

Además, Rai cuenta con un tejido de sociedades subsidiarias:

- Rai Pubblicità: Asume la programación publicitaria del sistema público.

- Rai Com: Encargada de la distribución de permisos y derechos de transmisión audiovisual de los contenidos de Rai en todo el mundo.

- Rai Way: Concesionaria de las estaciones base y de la difusión de señal de televisión en Italia.

- Rai Cinema: Gestiona la compra de derechos de películas, nacionales e internacionales, para las cadenas de Rai.

\subsection{Mandato de servicio público y rendición de cuentas}

El actual papel que ha de desempeñar la radiotelevisión pública en el marco de la sociedad italiana está perfectamente explicitado en un Decreto del gobierno de Paolo Gentiloni publicado en 2017 (Gazzetta Ufficiale, número 118) en la que se establece que Rai se configura como una concesionaria en exclusiva del "servicio público televisivo, radiofónico y multimedia". Este estatus, confirmado en marzo de 2018 tras la publicación del contrato Estado-Servicio Público, indica además que dicha concesión se extiende a lo largo de diez años.

Al margen de las guías básicas que se establecen para los medios públicos europeos, donde la información de calidad, el entretenimiento y la divulgación son las premisas básicas, el sistema italiano ahonda en el rol plural, imparcial, transparente y veraz de los contenidos informativos. Sin embargo, se incluyen también algunas prioridades técnicas pues se confiere a Rai la misión de poner en marcha servicios interactivos digitales de utilidad pública.

No faltan, en cualquier caso, referencias en este Decreto que aluden al esfuerzo que debe desplegar Rai para evitar contenidos perniciosos, proteger los derechos de los menores, proyectar una imagen no estereotipada de la mujer, fortalecer a las minorías y trabajar por la inclusión y diversidad.

Con el fin de concretar a ojos del escrutinio público estas cuestiones, Rai está obligada a dar a conocer un "balance social" antes del cierre de cada año y en 


\section{Las nuevas narrativas, en el entorno social}

Universidad de La Laguna, diciembre de 2019

paralelo al balance de resultados económicos. Se trata, a todas luces, de un esfuerzo por la transparencia en consonancia con las exigencias a las que ha de hacer frente un medio público en el siglo XXI.

Según establece el Decreto referido, la Rai está sometida a la vigilancia de dos instituciones clave y específicas en relación con la misión del audiovisual público italiano:

- La Autorità per le Garantie nelle Comunicazioni, que desempeña la labor de organismo regulador.

- Ministerio de Desarrollo Económico.

Interesante cuestión esta si tenemos en cuenta que, en el plano económico en tanto que accionista principal de Rai es imprescindible la figura del Ministerio de Economía y Finanzas.

\subsection{La oferta de servicios digitales de Rai}

La gran apuesta de la radiotelevisión pública italiana en el ámbito digital se centra en la extensión de la plataforma en internet.

Según afirma su balance anual de 2018, Rai ha ido progresivamente mejorando y enriqueciendo su oferta, donde el principal canal es RaiPlay.it (https://www.raiplay.it). Desde esta plataforma, los usuarios acceden a la programación de las distintas cadenas de televisión en streaming y a algunos de los programas bajo demanda. En paralelo -y renovada profundamente entre 2017 y 2018-, esencial es también la web para los canales radiofónicos públicos: Raiplay Radio (https://www.raiplayradio.it/).

Con peso fundamentalmente informativo, y replicando la propuesta de los telediarios y servicios de noticias de Rai, ocupa también un lugar destacado RaiNews (http://www.rainews.it).

La oferta en internet se complementa con RaiPlay Yoyo (https://www.raiplay.it/programmi/raiplayyoyo/), de reciente creación y vinculada al público infantil, y la plataforma de contenidos exclusivos y dedicada a los eventos deportivos RaiSport (http://www.raisport.rai.it), donde el acceso a las noticias de este ámbito son el principal reclamo.

No hay que dejar de lado el portal corporativo de Rai (www.rai.it), escaparate para comprender la estructura de la radiotelevisión pública italiana, su 


\section{Las nuevas narrativas, en el entorno social}

Universidad de La Laguna, diciembre de 2019

información institucional y el acceso a los contenidos a través de los portales descritos.

En cualquier caso, conviene recordar que el cuidado y la difusión del patrimonio audiovisual italiano es uno de los objetivos de servicio público que constituyen la Misión de Rai. Por lo que, en este sentido, el acceso a parte de los contenidos históricos es posible gracias a estos canales. El resto, en continuo proceso de digitalización, suele estar disponible para consulta e investigación a través de distintas modalidades más allá de las extensiones web de la radiotelevisión pública italiana.

Asimismo, y como reflejo de la presencia editorial en internet de la oferta de Rai, esta ha trabajado para adecuar al ámbito de las aplicaciones en el móvil sus cuatro grandes apuestas: RaiPlay, Raiplay Radio, RaiNews y RaiPlay Yoyo.

En paralelo a estas propuestas, Rai no descuida su canal específico en YouTube, herramienta sobre la que el Balance de 2018 (p. 82) manifiesta que se ha convertido en una apuesta para hacer más extensivos y dotar de mayor alcance a los contenidos de la radiotelevisión pública italiana.

La siguiente meta en la que se está trabajando para complementar la apuesta digital de Rai en internet es un canal específico sobre información meteorológica y tráfico que, si bien está en desarrollo, aún no se ha concretado a cierre de ejercicio en 2018.

\subsection{Proyectos de innovación y desarrollo de nuevas tecnologías para mejorar su oferta de productos y servicios a los ciudadanos}

A juzgar por lo referido en el balance anual de cierre de 2018 de Rai, la actividad de la radiotelevisión pública italiana en el ámbito del I+D ha sido bastante intensa. Gran parte del empeño puramente tecnológico de este servicio audiovisual público se perfila como una exigencia en el ya mencionado Contrato Estado-Concesionaria que regula los objetivos de Rai.

Toda la labor de I+D de la pública italiana se dirige hacia dos metas bien señaladas:

1) Desarrollo de productos y oferta televisiva, así como de la infraestructura y estándares tecnológicos que ello requiera. 
2) Mejora de los procesos internos de Rai como empresa audiovisual.

Es interesante advertir que existe un Laboratorio Mercato Tecnologico, encargado de monitorizar algunos de los proyectos sobre los que está trabajando Rai, y que, en ocasiones, se ponen en marcha iniciativas que cuenta con el apoyo de otras instituciones como la Universidad de La Sapienza de Roma o los Politécnicos de Milán y Turín.

Dado que en una atmósfera de amplio cambio tecnológico el compromiso es el de mejorar constantemente la calidad técnica, muchos de los proyectos de I+D dinamizados por Rai ponen el acento en los nuevos formatos de alta definición y la transmisión de una señal que requiere cada vez más sofisticación. En este sentido, conviene subrayar el especial rol que asume Rai Way cuyo principal marco de acción es la difusión de señal y la continua mejora de las infraestructuras de transmisión. Precisamente, una de las principales innovaciones que se está corporeizando en la pública italiana es el sistema EVA, vital para una óptima difusión de la señal, sin descuidar la apuesta por la señal $\mathrm{DAB}+$, imprescindible para la implementación de la radio digital. Pero los esfuerzos por adaptar y actualizar la propuesta radiofónica de Rai a un futuro tecnológico cada vez más exigente y más cercano ha obligado a la radiotelevisión pública italiana a actualizar y probar un nuevo sistema que mida la calidad de la señal de las cadenas radiofónicas de Rai que se reciben en los teléfonos móviles a través de la aplicación oficial. Asimismo, también se están probando modelos de personalización que mejoran la experiencia de los usuarios 5 .

Las innovaciones más significativas que se proyectan para las cadenas televisivas se dirigen a adaptar los contenidos a los requerimientos de los estándares tecnológicos de alta calidad (HD, Full HD, UHD). Por ello, Rai está trabajando en estrategias de innovación tecnológica que, por un lado, tratan de pautar la evolución de los sistemas de producción de contenidos -en concreto de los formatos de ficción-; y por otro, el estudio y la distribución de servicios multiplataformas.

\footnotetext{
5 En concreto, se ha trabajado sobre la transmisión de óperas líricas y sinfónicas en
} Radio Rai. 


\section{Las nuevas narrativas, en el entorno social Universidad de La Laguna, diciembre de 2019}

El marco de operaciones, por tanto, se está concentrando en los sistemas de difusión-recepción de la señal a través de la tecnología IP, al mismo tiempo que se estudia y experimenta con la tecnología HDR o se dan pasos para adaptar los modelos de interactividad de la oferta de contenidos de Rai al nuevo estándar HbbTv. De todas estas innovaciones no quedan al margen los procesos de digitalización del ingente archivo de la radiotelevisión pública italiana.

Con el fin de tratar de entender con propuestas concretas el trabajo en innovación y desarrollo emprendido por Rai, señalamos a continuación algunos de los proyectos más tangibles emprendidos a lo largo de 2018:

- Pruebas y mejoras del contenido enriquecido en la oferta de televisión lineal.

- Plataforma de periodismo de datos. Se ha completado una cobertura específica sobre los feminicidios gracias a un marco de cooperación con la Universidad de Turín.

- Se están realizando pruebas para que los telespectadores de Rai puedan visualizar un programa en directo desde el inicio, aunque este haya ya comenzado.

- Servicio de televisión ralentizada que, al bajar la velocidad de emisión a petición del usuario, permite una mayor comprensión. Esta propuesta está dirigida a público anciano o con algunas dificultades sensoriales.

- Servicio de publicidad personalizada que se activará en las televisiones conectadas en la emisión de las interrupciones publicitarias de las cadenas Rai.

- Servicio de transcripción automática que mejorará las técnicas de subtitulado de contenidos.

- Aplicación de "hybrid radio" para personalizar en la aplicación oficial de Rai los programas preferidos por cada usuario.

- Aplicación que permitirá buscar 'podcasts' de Rai al dictar a los dispositivos palabras clave.

- Pruebas para definir un nuevo estándar vía satélite DVB para alertar de emergencias a la ciudadanía. 


\section{Las nuevas narrativas, en el entorno social}

Universidad de La Laguna, diciembre de 2019

- Desarrollar una equipación de drones para realizar tomas desde ópticas privilegiadas y disruptivas.

Más allá de estos ejemplos, Rai no puede perder margen de maniobra para el siguiente desafío: la reasignación de frecuencias de la televisión digital con el fin de dejar espacio para los servicios 5G. En este sentido, la I+D de la radiotelevisión pública italiana se reserva también para proyectos destinados a reorganizar los múltiplex de Rai.

Precisamente la futura implantación del $5 \mathrm{G}$ obligará también a las plataformas de televisión a estar preparadas para un nuevo contexto de mejoras tecnológicas y de calidad. Conscientes de esa necesidad, Rai ya está experimentando con la transmisión de la señal de los telediarios regionales para que sean compatibles con la señal $5 \mathrm{G}$. Asimismo, se están probando modelos de transmisión de la señal de televisión en directo hacia los móviles con el fin de minimizar los riesgos de retardo o mala calidad. En definitiva, la siguiente frontera será la distribución de contenidos $5 \mathrm{G}$.

Según especifica el balance económico de Rai en el ejercicio de 2018, para toda esta labor se destinaron 28,3 millones de euros ${ }^{6}$.

\section{Políticas sobre servicio público audiovisual y digitalización en Italia} A menudo se suele definir la política italiana como convulsa: gobiernos poco estables, escándalos y coaliciones imposibles han predominado en los últimos años en el país transalpino. En consonancia, la gestión de Rai y sus diferentes marcos normativos no han quedado exentos de polémicas ni libres de convertirse en arma arrojadiza política entre los distintos partidos.

Buen ejemplo de ello fue la mítica Ley Gasparri de 2004 que, como subrayamos al principio de este informe, se convirtió en un importante eje de controversia, incluso contestada por la Unión Europea. Durante el gobierno del socialdemócrata Romano Prodi (2006-2008), sin embargo, se quiso dar un giro de escena en este ámbito y lanzar una profunda reforma del sistema audiovisual público italiano. El encargado de esa misión fue el entonces

${ }^{6}$ Véase página 139 del informe de resultados económicos. 
ministro de Comunicaciones, Paolo Gentiloni ${ }^{7}$, quien en 2007 propuso un nuevo esquema en el que Rai estaría gestionada por una fundación que asumiría el rol de principal accionista difuminando los vínculos tan estrechos con el aparato estatal italiano. Asimismo, se diseñó un consejo de administración algo más amplio pero en el que se diluía el poder que ejercía la comisión parlamentaria que controlaba la gestión de la radiotelevisión pública previsto en la legislación anterior. Se instituía ahora un consejo compuesto por once miembros que se repartían de la siguiente manera: cuatro consejeros elegidos por la Comisión de Vigilancia Rai del Parlamento italiano -designados por mayoría de 2/3-; dos miembros elegidos por la confederación EstadoRegiones; uno designado por el Consejo Nacional de Economía y Trabajo; uno más propuesto por el Consejo Nacional de Consumidores y Usuario; un consejero por parte del Consejo Escolar -en el que se representan los institutos de secundaria y bachillerato-; otro miembro elegido por la confederación de Rectores de Universidades italianas, y por último, un miembro propuesto por la asamblea de trabajadores de Rai.

En cualquier caso, la precipitada caída del Ejecutivo de Prodi en 2008 bloqueó el avance parlamentario de la reforma impulsada por Gentiloni, por lo que durante varios años la radiotelevisión pública italiana se fue ajustando a los nuevos marcos y exigencias con la adaptación y la aprobación de determinados decretos puntuales.

Con el fin de aclarar mejor los cambios normativos que han readaptado el funcionamiento de Rai en Italia en función de la evolución política que ha vivido el país durante nuestro periodo de estudio, proponemos el siguiente cuadro resumen que aclara los principales hitos en este sentido:

\begin{tabular}{|l|l|l|l|}
\hline $\begin{array}{l}\text { Periodo de } \\
\text { mandato }\end{array}$ & Primer ministro & $\begin{array}{l}\text { Formación } \\
\text { política }\end{array}$ & Marco \\
& & & \\
\hline
\end{tabular}

${ }^{7}$ No conviene perder de vista que Gentiloni se convirtió en primer ministro nueve años después tras el fracaso de Matteo Renzi. 


\begin{tabular}{|c|c|c|c|}
\hline $\begin{array}{l}2013 \text { (año que } \\
\text { marca el final de } \\
\text { mandato) }\end{array}$ & Mario Monti & $\begin{array}{l}\text { Sin afiliación } \\
\text { política }^{8} \text {. }\end{array}$ & $\begin{array}{l}\text { Sin cambios } \\
\text { reseñables. }\end{array}$ \\
\hline 2013-2014 & Enrico Letta ${ }^{9}$ & & \\
\hline $2014-2016$ & Matteo Renzi & $\begin{array}{l}\text { Partido } \\
\text { Demócrata }\end{array}$ & $\begin{array}{l}\text { Entra en vigor la } \\
\text { nueva legislación } \\
\text { específica para } \\
\text { Rai (ley número } \\
220 \text { del } 28 \text { de } \\
\text { diciembre de } \\
2015 \text { ) }\end{array}$ \\
\hline 2016- 2018 & Paolo Gentiloni & $\begin{array}{l}\text { Partido } \\
\text { Demócrata }\end{array}$ & $\begin{array}{l}\text { En 2017, y tras la } \\
\text { aprobación de la } \\
\text { nueva normativa } \\
\text { de Rai, entran en } \\
\text { vigor dos } \\
\text { decretos: }\end{array}$ \\
\hline
\end{tabular}

${ }^{8}$ Monti fue designado primer ministro por decisión del presidente de la República, Giorgio Napolitano, tras la dimisión precipitada de Silvio Berlusconi. Como primer ministro, el gran desafío de Monti fue lidiar con un país inmerso en una honda crisis de deuda. Tras su mandato, decidió presentarse a los siguientes comicios con un partido de nuevo cuño liderado por él mismo de corte liberal: Elección Cívica.

9 Letta gobernó en coalición con el derechista Popolo della Libertà, partido liderado en su día por Silvio Berlusconi. La inestabilidad que vivía el país y los acuerdos de coalición no hicieron popular a Letta dentro de las filas del Partido Demócrata, hecho que provocó su sustitución por Matteo Renzi. 


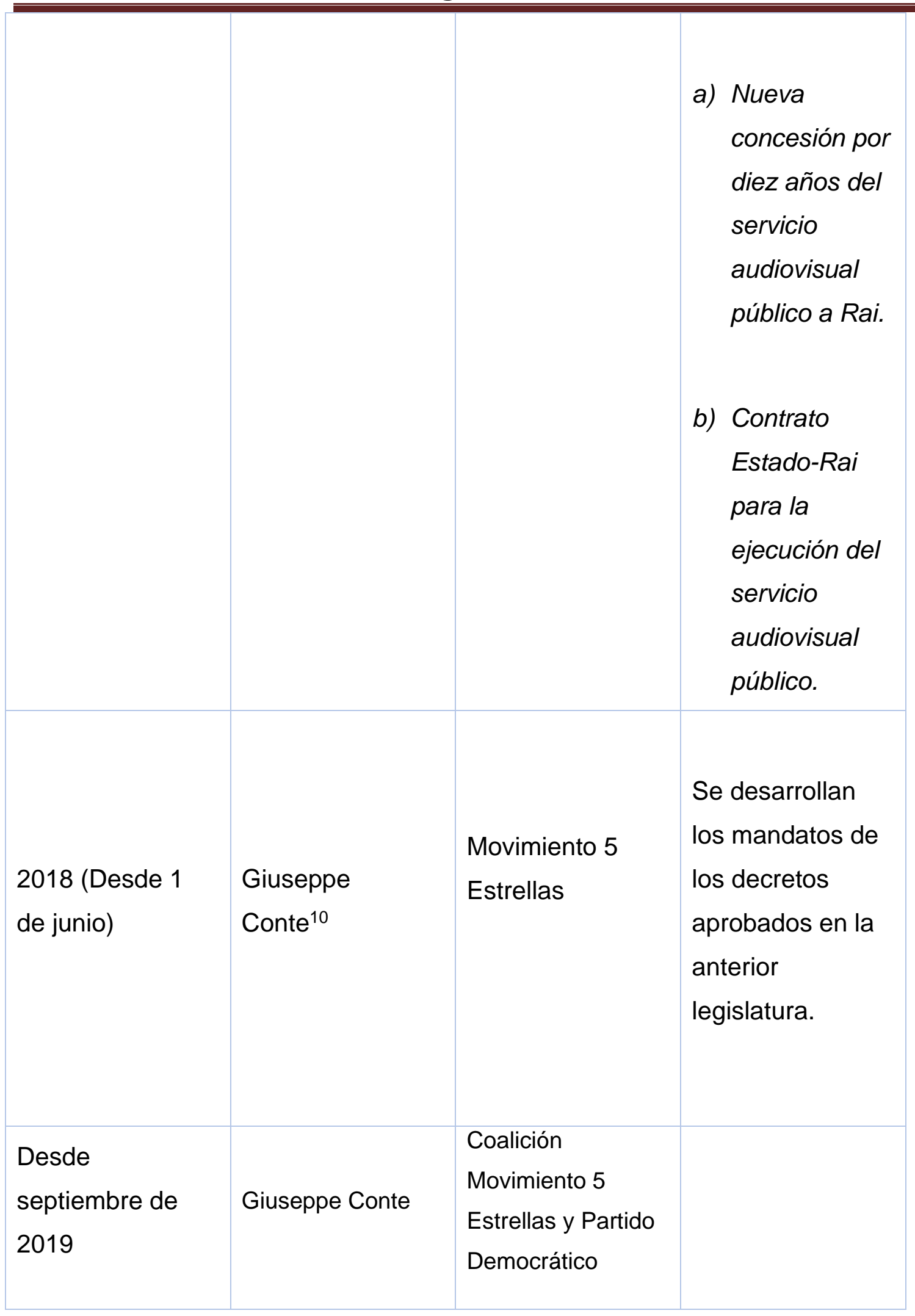

${ }^{10}$ Conte es primer ministro por el acuerdo de la coalición de gobierno firmada por el derechista y nacionalista Liga, partido dirigido por Matteo Salvini, y el Movimiento 5 Estrellas, habitualmente considerado como partido de izquierda antisistema, cuyo líder es Luigi di Maio. 


\section{Las nuevas narrativas, en el entorno social \\ Universidad de La Laguna, diciembre de 2019}

Como vemos en el cuadro resumen, el principal salto cualitativo en cuanto al marco legislativo de la radiotelevisión pública italiana se da en 2015. La citada ley 220 de 28 de diciembre de aquel año supuso toda una renovación en el eje corporativo y de administración de Rai.

El texto instituye la figura del consejero delegado cuyo mandato se prolongará durante tres años cuya designación corresponderá al consejo de administración bajo propuesta de la Asamblea de Socios que, en definitiva, corresponde en Italia al Ministerio de Economía ${ }^{11}$. Entre sus competencias, destaca la de asumir el día a día de la gestión de la radiotelevisión pública, así como elegir a los directores de las distintas unidades, aunque, cuando se trata de la designación de los máximos responsables de las distintas cadenas, el consejero delegado debe consultar al consejo de administración y obtener su apoyo.

En paralelo, la Ley activa el rol del 'presidente di garanzia', con funciones no ejecutivas, pero clave a la hora de verificar si Rai cumple su compromiso de servicio público. Uno de los miembros del consejo de administración asumirá este rol. Precisamente, con respecto al consejo de administración se mantiene una estructura compuesta por siete miembros, aunque se redimensiona su origen desperfilando el peso definitivo del Parlamento en este ámbito. De esos siete miembros, cuatro los elegirá la Cámara italiana a razón de mayoría de 2/3 a través de la comisión específica que aborda los asuntos del servicio audiovisual público. Dos de los siete consejeros los elegirá el Gobierno. El consejero que resta vendrá determinado por el consejo de los trabajadores de Rai. Entre otros aspectos, se introducen cláusulas que velan en especial por la transparencia del modelo, sobre todo cuando se refiere a contratos públicos por encima de los 200.000 euros. Eso sí, la salvedad queda hecha cuando este tipo de contratos, y por esta cuantía, se refiere a los de las figuras estrellas de la programación de Rai.

\footnotetext{
${ }^{11}$ No en vano, Rai sigue instituyéndose como una sociedad por acciones de la que el Estado es su accionista principal y único.
} 
Una vez fijada la nueva normativa, en 2017 se reajusta la misión de servicio público de Rai. Sobre todo, porque ya caducaba la anterior concesión pública. Con el nuevo decreto se amplía la dimensión de la misión de Rai para el país en tanto que se refuerzan las políticas que exigen que la radiotelevisión pública italiana dé cabida en sus contenidos a propuestas paritarias para reforzar el papel de la mujer y a aspectos que reflejen las prioridades/necesidades de las distintas minorías.

Además, como ya señalamos en la parte anterior de este informe, la digitalización de infraestructuras y contenidos se convierte, asimismo, en otra exigencia para Rai a partir de la publicación en 2017 del marco de la nueva concesión y de la confirmación de esta a través del contrato entre Estado y radiotelevisión pública.

Así las cosas, el anterior ejecutivo de coalición (Lega + Movimiento 5 Estrellas) liderado por Giuseppe Conte, se ha limitado a aplicar el marco legislativo que heredó de Paolo Gentiloni. Como al anterior presidente del Partido Demócrata solo le dio tiempo a firmar los decretos que marcan las prioridades de Rai para los próximos diez años, el Ejecutivo de Conte se ha encontrado con todo el trabajo legislativo hecho. A él le corresponde llenar de contenido y determinar los nombramientos de aquellos que liderarán Rai en los próximos años.

Del mismo modo, el gobierno actual que lidera Conte deberá ejecutar el siguiente desafío normativo y técnico al que tiene que hacer frente la televisión italiana: la reasignación de frecuencias y la preparación para incorporar el 5G. En este sentido, la legislación de referencia -aprobada por el Gobierno anterior en 2015 y matizada posteriormente en consonancia con la decisión de la Unión Europea 2017/899- ha estado sometida a consulta pública para clarificar y cimentar lo que en Italia se ha denominado Plan Nacional de Reparto de Frecuencias ${ }^{12}$.

En este contexto, la principal prioridad que se ha marcado Roma es la de reasignar las frecuencias de la banda 694-790 Mhz en los próximos cuatro años (2018-2022). Estas frecuencias son las que están destinadas a la

\footnotetext{
12 Puede consultarse el estatus de este proceso en esta comunicación oficial del Ministerio de Desarrollo Económico:

https://www.mise.gov.it/images/stories/documenti/sintesi\%20consultazione\%20PNRF \%2018.pdf
}

ISBN: 978-84-17314-22-4 / D.L.: TF 148-2020 / DOI del libro: 10.4185/CAC166

Página | 239 
transmisión de televisión nacional y local. Se trabaja para que los canales aquí contenidos puedan trasvasarse a sistemas terrestres para que estas bandas liberadas puedan ofrecer servicios de comunicación electrónica y banda ancha inalámbrica con el fin, y de manera esencial, de poder poner en marcha la tecnología 5G.

Obviamente, todo este trabajo conlleva la reasignación de los canales de la televisión digital terrestre y, además, habilitar las frecuencias en la banda III VHF para que se pueda desarrollar un múltiplex regional de televisión local que aumente la oferta de contenidos. Gracias a esta reasignación, el Gobierno italiano busca, por otra parte, optimizar los bloques que puedan asignarse a la radio digital (DAB) al mismo tiempo que se pretende reforzar la prioridad del sistema público italiano en cuanto a información regional y programación local. El plazo marcado para asumir todo este proceso comprende desde el 1 de enero de 2020 hasta el 30 de junio de 2022.

El principal garante de esta transformación es el Ministerio de Desarrollo Económico (Ministero per lo Sviluppo Economico). Se trata de un departamento ministerial de mucho peso y poder bajo cuyo control, además de las políticas de comunicaciones, se aglutinan las áreas de energía, comercio, mercado, consumidores y usuarios y sistemas de apoyo y subvenciones. Como refiere Pacino (2018) la coalición de gobierno italiana preveía una lucha de contrapesos entre los dos partidos implicados en ese momento: Lega -hoy desaparecido del gobierno- y Movimiento 5 Estrellas. El objetivo era tratar de compensar el poder de un ministro de un partido, con toda una sucesión de subsecretarios de la otra formación con el fin de establecer equilibrios no siempre sencillos.

En el ámbito de las Comunicaciones que gestiona este gran Ministerio, los sectores sobre los que se actúa son los siguientes: Radio, Televisión, Correos, Telefonía, Internet y Banda Ancha entre otras áreas auxiliares.

A lo largo de este trabajo ya hemos señalado cómo la apuesta hacia la televisión del futuro condiciona gran parte del compromiso de servicio público y evolución técnica de Rai. No en vano, los trabajos dirigidos por este Ministerio para readaptar la TDT y facilitar la llegada del $5 G$ se ven complementados con 


\section{Las nuevas narrativas, en el entorno social}

Universidad de La Laguna, diciembre de 2019

el objetivo de implantar la televisión 4.0, misión para la que están preparando mesas específicas de trabajo.

No hay que olvidar, en cualquier caso, que el organismo regulador para este tipo de políticas que afectan a la televisión (y a otros medios) es la Agcom, Agencia para las Garantías de las Comunicaciones (Agenzia per le Garanzie delle Comunicazioni), que en muchas ocasiones funciona como árbitro y en otras como asesor técnico imprescindible.

\section{Conclusiones}

- El desarrollo y la consolidación de la TDT ha permitido ampliar el espectro de Rai en contenidos y posibilidades técnicas. De un modelo de tres cadenas de televisión generalistas y tres cadenas de radio análogas se ha pasado a un gigante televisivo que diversifica y atiende a públicos concretos.

- Del mismo modo que se ha crecido en contenidos, Rai ha experimentado un interesante desarrollo en formatos y propuestas digitales, aunque conviene reseñar que, en este sentido, las plataformas privadas y de pago superan en audiencia y alcance a las públicas.

- La proyección digital de los contenidos Rai a través de redes sociales, aplicaciones y plataformas como YouTube se ha convertido en un elemento esencial para visibilizar sus propuestas de contenidos. Incluso, con vistas a la promoción exterior de estos aunque, por experiencia propia de este grupo de investigadores, hemos notado que la mayor parte de los programas y la emisión en directo de contenidos de entretenimiento está vedada fuera de las fronteras italianas. Se percibe, por tanto, un celo especialmente restrictivo por parte de Rai a la hora de explotar los derechos en el exterior de sus contenidos audiovisuales. En la práctica es un ejemplo de "geoblocking", cuestión que puede confrontar con la misión impulsada por la Comisión Europea del mercado único digital.

- Interesante es la acción de Rai en materia de innovación, desarrollo e investigación. Si bien es una parte clave de su misión como servicio público que es, percibimos que en la radiotelevisión pública italiana se trabaja para 


\section{Las nuevas narrativas, en el entorno social}

Universidad de La Laguna, diciembre de 2019

no quedarse atrás en materia de digitalización y adaptación de las infraestructuras a las nuevas redes y a los nuevos contenidos que vendrán de la mano del 5G. Los trabajos que se están desarrollando hoy día en este sentido están centrándose en verificaciones y pruebas, aunque alguna experiencia concreta se ha podido ir materializando tal y como hemos visto en el segundo punto de este trabajo.

- El principal riesgo al que se ve sometida Rai es a la indeterminación y la interferencia políticas. La inestabilidad y la lucha partidista en muchas ocasiones contamina la misión de servicio público de la radiotelevisión italiana en varios frentes:

- La designación de directivos en áreas de responsabilidad y dirección de cadenas

- La propuesta editorial

- La confusión técnica

Pese a todo, si bien el anterior gobierno en coalición que lideró el país hasta el pasado mes de septiembre ha dado mucho que hablar a la hora de configurar el organigrama de Rai tomando como base la normativa de 2017, el Ejecutivo ha sido más eficaz a la hora de proseguir con los elementos técnicos que no pueden paralizarse en función del cumplimiento con el marco europeo. Nos referimos en concreto a que, desde el Ministerio específico, el de Desarrollo Económico, no se están paralizando los trabajos ya iniciados en la anterior legislatura para adaptar las frecuencias y las infraestructuras a la televisión del futuro y a la llegada del $5 \mathrm{G}$.

\section{Referencias bibliográficas}

A Biondi (2018): "Netflix \& Co raddoppiano gli utenti Boom da 4 a 8 milioni in un anno" en II Sole 24 Ore, diario de información económica. Milán. Publicado el 22 de noviembre de 2018.: https://www.ilsole24ore.com/art/notizie/2018-1122/netflix-co-raddoppiano-utenti-boom-4-8-milioni-unanno-

071209.shtml?uuid=AEhByukG\&refresh ce=1 (consultado el 12 de septiembre de 2019).

Comisión Europea (2006) : «Competition: Commission requests Italy to comply with EU rules on electronic communications », Nota de prensa, Bruselas, 19 de 


\section{Las nuevas narrativas, en el entorno social \\ Universidad de La Laguna, diciembre de 2019}

julio.: $\quad$ http://europa.eu/rapid/press-release IP-061019 en.htm?locale=en (consultado en 12 de septiembre de 2019).

Confindustria Radio Televisioni (2017) : «Studio Economico Settore Televisivo Privato Italiano », $23^{\circ}$ Edizione, Osservatorio Nazionale delle Imprese Radiotelevisive Private,

Roma: http://confindustriaradiotv.it/wpcontent/uploads/2018/01/Studio\%20Economico \%20TV\%202017.pdf (consultado el 1 de octubre de 2019).

Gazzetta Ufficiale del Parlamento Italiano:

- 2001, Conversione in legge, con modificazioni, del decreto-legge 23 gennaio 2001, n. 5, recante disposizioni urgenti per il differimento di termini in materia di trasmissioni radiotelevisive analogiche e digitali, nonché per il risanamento di impianti radiotelevisivi, Legge $n$ 66, 20 de marzo. Publicada en la Gaceta Oficial el día 24 de mayo en el Supplemento Ordinario, ํㅜ http://www.parlamento.it/parlam/leggi/01066l.htm (consultado el $15 \mathrm{de}$ octubre de 2-019)

- 2004, Norme di principio in materia di assetto del sistema radiotelevisivo e della RAI Radiotelevisione italiana spa, non cehé delega al Governo per l'emanazione del testo único della radiotelevisione, Legge n 112, 3 maggio. Publicada en la Gaceta Oficial el día 5 de mayo en el Supplemento Ordinario, no 82.: http://www.camera.it/parlam/leggi/04112l.htm (consultado el 15 de octubre de 2019).

Gazzetta Ufficiale della Repubblica Italiana:

- 2017, Affidamento in concessione del servizio pubblico radiofonico, televisivo e multimediale ed approvazione dell'annesso schema di convenzione, decreto. Publicado el 23 de mayo de 2017 en la Serie General de esta Gaceta, número 118: http://www.gazzettaufficiale.it/eli/gu/2017/05/23/118/sg/pdf (consultado el 10 de octubre de 2019).

- 2018, Contratto di servizio Rai 2018-2022, número 55, publicado el 7 de marzo: https://www.mise.gov.it/images/stories/documenti/Contratto-diservizio-Rai-2018-2022.pdf (consultado el 10 de octubre de 2019). 
Ministerio dello Sviluppo Economico (MISE) :

- 2017, Convenzione fra il Ministero dello sviluppo economico e la RAI per la concessione per il servizio pubblico radiofonico, televisivo e multimediale, 27 de julio, Roma: https://www.mise.gov.it/images/stories/documenti/convenzione mise rai 27 luglio 2017.pdf (consultado el 10 de octubre de 2019).

G. Pacino (2018) : "Braccio di ferro Di Maio-Salvini sul dossier Comunicazioni", CorCom, Milán, 5 de junio: https://www.corrierecomunicazioni.it/digitaleconomy/delega-alle-tlc-braccio-di-ferro-di-maio-salvini// (consultado el 15 de octubre de 2019).

Rai - Radiotelevisione Italiana :

- 2015, Statuto: http://www.rai.it/dl/doc/1533290900193 Statuto.pdf (consultado el 12 de septiembre de 2019).

- 2018, Bilanci separato e consolidato intermedi al 30 giugno 2018, Roma: http://www.rai.it/dl/doc/1542206415179 Rai\%20Semestrale\%202018\%2 09.11.2018.pdf (consultado el 12 de septiembre de 2019).

M. Scaglioni (2016) : Il servicio pubblico televisivo. Morte o rinascita della Rai ?. Milán : Vita e pensiero.

E. Viggiani (2008): "La TV digitale terrestre in Italia: dalle parole ai atti", conferencia en Oviedo, 6 de noviembre: http://www.camaraovi.es/documentos/informatica/TDT\%20DGTVi\%20\%20Edigio\%20Viggiani.pdf (consultado el 12 de septiembre de 2019).

* Este artículo se ha realizado dentro del Proyecto CSO2017-82277-R, LoS retos de la televisión pública en España ante el mercado único digital europeo: estrategias multipantalla, innovación y renovación de los mandatos del servicio público, financiado por el Ministerio de Ciencia, Innovación y Universidades. 